\title{
Multisystem Inflammatory Syndrome in Children in COVID-19 Pandemic
}

\author{
Satish K. Shah ${ }^{1}$ - Alvaro Coronado Munoz ${ }^{1}$
}

Received: 19 June 2020 / Accepted: 25 June 2020 / Published online: 3 July 2020

(C) Dr. K C Chaudhuri Foundation 2020

The Multisystem System Inflammatory Syndrome in Children (MIS-C) has recently been described during COVID-19 pandemic. It has gained considerable interest for few reasons. First, it tends to manifest later in the course of SARS-CoV-2 infection and presents with higher severity. Second, even though it shares clinical and laboratory features of Kawasaki disease and Kawasaki-shock syndrome, the demographic and inflammatory process are different from the classical description.

The case reported in this issue of Indian Journal of Pediatrics by Rauf et al. [1] describes the classical presentation of MIS-C which shares features similar to Kawasaki Disease. The authors report a 5-y-old boy from a COVID-19 hotspot area in India who presented with high grade fever, abdominal pain, diarrhea, bulbar conjunctivitis, edema and shock. Patient had increased C-reactive protein (CRP), ESR and ferritin suggesting a hyperinflammatory state. An elevated pro-BNP suggested acute heart failure confirmed by left ventricular hypokinesia and depressed left ventricular function on echocardiogram. An elevated Troponin-I indicated myocardial injury; however, the patient was not found to have coronary artery enlargement. Patient was managed with Intravenous Immunoglobin (IVIg) and pulse dose methyl prednisolone. Adrenaline was used for hypotension while diuretics and afterload reduction were used for acute heart failure. A repeat echocardiogram showed improved biventricular function. On day six of hospital stay, the patient was able to be discharged home with low dose aspirin, steroid and enalapril. Patient had initially tested negative for SARS-CoV-2 by RT- PCR and

Satish K. Shah

satish.k.shah@uth.tmc.edu

1 Department of Pediatrics, Pediatric Critical Care Division, University of Texas- Health Science Center at Houston, Children's Memorial Hermann Hospital, Houston, TX 77030, USA antibody testing was unavailable. However, there was history of possible COVID exposure in patient's father.

In the global pandemic setting of COVID-19, there are relatively fewer cases of COVID-19 children compared to adults. Children tend to have milder clinical course. Several reports published from different parts of the world have confirmed that severe illness and death due to COVID-19 in children is rare and is much less compared to adults [2-6]. However, in last $6 \mathrm{wk}$, especially at the end of peak number of cases in the developed world, pediatricians have started seeing multisystem inflammatory disorder similar to Kawasaki disease or Toxic Shock syndrome [7-9]. Children presented in critical conditions with heterogeneous inflammatory involvement. Various reports from many countries of children with fever and inflammation without obvious cause in the time of COVID-19 pandemic hints toward possible link between SARS-CoV-2 and this clinical syndrome. With the rise in similar case presentations, a case definition for Multisystem Inflammatory Syndrome in Children was proposed by the World Health Organization, the UK-based European Center for Disease Prevention and Control, and the US-based center for Disease Control and Prevention.

MIS-C is rare but with common characteristics that have been observed during the COVID-19 pandemic reported from different cohorts [7-12]. This disorder manifests with a wide spectrum of signs and symptoms. Fever is invariably present in most cases. Abdominal pain, diarrhea, and vomiting mimicking acute abdomen are the most striking symptoms of the clinical syndrome. Cardiovascular dysfunction and myocardial injury are present in more than half of the reported cases. Mucous membrane changes, conjunctival injection, swollen extremities and lymphadenopathy are also common, further mimicking Kawasaki disease. Notable laboratory features consist of anemia, increased neutrophils, low lymphocytes with normal to low platelet count. An increased CRP and ferritin are seen invariably in most cases, suggesting a hyperinflammatory state. Increased D-dimer and fibrinogen level also suggest activation of the coagulation cascade and 
a prothrombotic state. Elevated Pro-BNP and troponin are often seen with cases involving cardiovascular system. Abdominal imaging shows evidence of bowel inflammation, gall bladder edema and lymphadenopathy. Echocardiogram may show left ventricular dysfunction and, less commonly, coronary artery dilatation. Most cases have undetectable SARS-CoV-2 on RT-PCR and only few cases have IgG antibody for SARS-CoV-2 virus. These findings probably suggest that this syndrome is a late inflammatory process within $4 \mathrm{wk}$ of an acute disease or contact with a COVID-19 person. Since the condition is evolving and true pathophysiology is not yet known, it is recommended to evaluate for other infectious entities such as adenovirus, coxsackie virus, EBV, mycoplasma, typhus, and parvovirus based on endemicity and availability of testing. The utility of these tests is to rule out other causes as well as delineate the therapies. Based on above mentioned clinical features and laboratory findings, it is thought that MIS-C shares a common etiology and pathophysiology with Kawasaki disease. Based on various reports that have been published, clinical signs and symptoms overlap with Kawasaki disease, but most do not meet clinical criteria required for diagnosis by the American Heart Association. There are more similarities and few differences between MIS-C and Kawasaki disease. Similar characteristics include prolonged fever, multisystem inflammation, mucocutaneous involvement, diarrhea, lymphadenopathy and high levels of inflammatory markers. Kawasaki disease is common in children less than 5-y-old whereas MIS-C is more common in children older than $5 \mathrm{y}$ of age. Left ventricular dysfunction is significantly more common in MIS-C compared to Kawasaki disease. Another difference has been the lack of reports of MIS-C from Asian countries where typical Kawasaki is more common. This observation has raised the global research question as to whether SARS-CoV-2 directly triggers MIS-C and Kawasaki disease like illness and if so, what factors determine the variations in the clinical symptoms.

Despite differences in the clinical spectrum, outcome data of the patients with this syndrome is promising in the developed world. Most patients diagnosed with this syndrome recover quickly if appropriate therapy is instituted early in the course of illness. Patients should ideally be managed in high dependency unit or intensive care unit for close monitoring, particularly if there is cardiovascular involvement. Patient may require inotropic support. The early recognition is going to help preventing the management of these patients with aggressive fluid resuscitation that can push the starling curve to a fulminant heart failure. In cases of severe cardiovascular dysfunction, ECMO therapy may be needed as a bridge to recovery if such methods are available. Immunomodulator therapy, particularly IV Immunoglobulin (IVIg) can be used as a first line treatment due to hyperinflammatory state and similarity in presentation to Kawasaki disease. The possibility of decreasing coronary involvement with this therapy is yet to be determined. This syndrome seems to have a higher number of patients with refractory IVIg response, often requiring a second dose. Regular dose or pulse methyl prednisolone can be added as second line therapy in the severe cases or in cases where inflammation persists despite IVIg. Few centers have reported successful use of IL-1 receptor antagonist (Anakinra) and TNF- $a$ antagonist (Infliximab) in the severe cases. Other therapies like IL-6 antagonist (Tocilizumab) can be used in severe illness which continues to worsen despite IVIg, steroid and anakinra. Another important consideration in this syndrome is hypercoagulability. Aspirin is typically used for coronary aneurysm and it should be considered in patients that meet Kawasaki syndrome phenotype. If there is thrombocytopenia, aspirin treatment should be avoided. In patients with severe ventricular dysfunction, treatment with heparin should be started as soon as possible. For other patients, prophylactic dosing of low molecular heparin is recommended due to elevated risk of thromboembolic event.

Multisystem Inflammatory Syndrome in Children (MIS-C) is a new syndrome in the evolving spectrum of COVID-19 pediatric illness that is typically experienced later during the course. It is a rare but serious condition requiring hospital admission and fine management. As there are increasing cases of COVID-19 in India, pediatrician should be acutely aware of this new clinical syndrome related to SARS-CoV-2 infection that overlaps with signs and symptoms of Kawasaki disease. Clinicians should operate with an increased index of suspicion and follow the case definition proposed by WHO and CDC to identify this syndrome early in the course of illness. Prompt identification with employment of appropriate therapies is the key to favorable outcomes.

\section{Compliance with Ethical Standards}

Conflict of Interest None.

\section{References}

1. Rauf A, Vijayan A, John ST, Krishnan R, Latheef A. Multisystem inflammatory syndrome with features of atypical Kawasaki disease during COVID-19 pandemic. Indian J Pediatr. 2020. https://doi. org/10.1007/s12098-020-03357-1.

2. Dong Y, Mo X, Hu Y, et al. Epidemiological characteristics of 2143 pediatric patients with 2019 coronavirus disease in China. Pediatrics. 2020;145:e20200702. https://doi.org/10.1542/peds. 2020-0702.

3. Lu X, Zhang L, Du H, et al. SARS-CoV-2 infection in children. New Engl J Med. 2020;382:1663-5.

4. Coronado Munoz A, Nawaratne U, McMann D, Ellsworth M, Meliones J, Boukas K. Late-onset neonatal sepsis in a patient with Covid-19. New Engl J Med. 2020;382:e49.

5. Shekerdemian LS, Mahmood NR, Wolfe KK, et al. Review of "characteristics and outcomes of children with coronavirus disease 2019 (COVID-19) infection admitted to US and Canadian pediatric 
intensive care units". JAMA Pediatr. 2020. https://doi.org/10.1001/ jamapediatrics.2020.1948.

6. González-Dambrauskas S, Vásquez-Hoyos P, Camporesi A, et al. Pediatric critical care and COVID19. Pediatrics. 2020:e20201766. https://doi.org/10.1542/peds.2020-1766.

7. Viner RM, Whittaker E. Kawasaki-like disease: emerging complication during the COVID-19 pandemic. Lancet. 2020;395:1741-3.

8. Riphagen S, Gomez X, Gonzalez-Martinez C, Wilkinson N, Theocharis P. Hyperinflammatory shock in children during COVID-19 pandemic. Lancet. 2020;395:1607-8.

9. Jones VG, Mills M, Suarez D, et al. COVID-19 and Kawasaki disease: novel virus and novel case. Hosp Pediatr. 2020;10:537-40.

10. Whittaker E, Bamford A, Kenny J, et al. Clinical characteristics of 58 children with a pediatric inflammatory multisystem syndrome temporally associated with SARS-CoV-2. JAMA. 2020. https://doi. org/10.1001/jama.2020.10369.

11. Verdoni L, Mazza A, Gervasoni A, et al. An outbreak of severe Kawasaki-like disease at the Italian epicentre of the SARS-CoV-2 epidemic: an observational cohort study. Lancet. 2020;395:1771-8.

12. Belhadjer Z, Méot M, Bajolle F, et al. Acute heart failure in multisystem inflammatory syndrome in children (MIS-C) in the context of global SARS-CoV-2 pandemic. Circulation. 2020. https://doi. org/10.1161/CIRCULATIONAHA.120.048360.

Publisher's Note Springer Nature remains neutral with regard to jurisdictional claims in published maps and institutional affiliations. 